\title{
Many-Body Localization with Long-Range Interactions
}

\author{
Rahul M. Nandkishore ${ }^{1}$ and S. L. Sondhi ${ }^{2}$ \\ ${ }^{1}$ Department of Physics and Center for Theory of Quantum Matter, University of Colorado at Boulder, \\ Boulder, Colorado 80309, USA \\ ${ }^{2}$ Department of Physics, Princeton University, Princeton, New Jersey 08544, USA \\ (Received 2 June 2017; revised manuscript received 14 August 2017; published 25 October 2017)
}

\begin{abstract}
Many-body localization (MBL) has emerged as a powerful paradigm for understanding nonequilibrium quantum dynamics. Folklore based on perturbative arguments holds that MBL arises only in systems with short-range interactions. Here, we advance nonperturbative arguments indicating that MBL can arise in systems with long-range (Coulomb) interactions, through a mechanism we dub "order enabled localization." In particular, we show using bosonization that MBL can arise in one-dimensional systems with $\sim r$ interactions, a problem that exhibits charge confinement. We also argue that (through the Anderson-Higgs mechanism) MBL can arise in two-dimensional systems with $\log r$ interactions, and speculate that our arguments may even extend to three-dimensional systems with $1 / r$ interactions. Our arguments are asymptotic (i.e., valid up to rare region corrections), yet they open the door to investigation of MBL physics in a wide array of long-range interacting systems where such physics was previously believed not to arise.
\end{abstract}

DOI: 10.1103/PhysRevX.7.041021

Subject Areas: Condensed Matter Physics, Statistical Physics

\section{INTRODUCTION}

The phenomenon of many-body localization (MBL) has drawn enormous interest from both the theory [1-8] and experimental [9-12] communities. The intensive investigation of the phenomenon has revealed a cornucopia of exotic physics, including connections to integrability [13-19], unusual response properties [20,21], a rich pattern of quantum entanglement [22-26], and new types of order that cannot arise in equilibrium [27-29]. MBL can also prevent heating in periodically driven Floquet systems [30-34] and thus protect new phases of driven quantum matter [35-40]. MBL has thus emerged as a powerful new paradigm for nonequilibrium quantum dynamics. However, at the same time there has been a proliferation of no-go arguments [41-50] constraining the settings in which MBL can arise. One of the most constraining of these is the restriction [1,51-53] to systems with short-range interactions.

The argument against MBL in systems with long-range interactions proceeds by examining the convergence of a perturbative locator expansion in $d$-dimensional systems when the Hamiltonian contains terms that decay as a power-law function of distance $\sim r^{-\alpha}$. An old argument due to Anderson [1] establishes that hopping terms with

Published by the American Physical Society under the terms of the Creative Commons Attribution 4.0 International license. Further distribution of this work must maintain attribution to the author(s) and the published article's title, journal citation, and DOI. $\alpha<d$ lead to the breakdown of the locator expansion (see also Ref. [51]). A refinement of this argument [52,53] establishes that two-body interactions with $\alpha<2 d$ also break the perturbative expansion. Based on these results, a folk theorem has arisen that holds that MBL cannot arise in systems with interactions longer ranged than $1 / r^{2 d}$. This excludes a great many experimentally relevant systems, including systems of charges (interacting with a Coulomb interaction in any dimension), and systems with dipolar $\left(1 / r^{3}\right)$ interactions in two and three dimensions. However, this folk theorem rests on shaky foundations, since the breakdown of the perturbation theory does not establish the breakdown of localization. Intriguingly, recent experiments [38] with dipoles seem to indicate MBL-type physics in a setting where this folk theorem would suggest such physics cannot arise. Could MBL survive after all in systems with long-range interactions?

In this work we present nonperturbative arguments indicating that MBL can arise with long-range interactions. Our conclusions apply even to interactions longer ranged than $1 / r^{d}$. The key idea is that the long-range interactions can drive the system into a nontrivial correlated phase, naturally described in terms of emergent degrees of freedom with only short-range interactions. The problem can then be mapped onto the classic analysis of Ref. [3] to establish many-body localization. We dub this mechanism "order enabled localization." We demonstrate the viability of this idea, using nonperturbative techniques to treat the interaction, for Coulomb interacting systems in any dimension (i.e., one-dimensional systems with $\sim r$ interactions, two-dimensional systems with $\log r$ interactions, 
and three-dimensional systems with $1 / r$ interactions). The arguments are presented in decreasing order of rigor, with the one-dimensional analysis being on the firmest footing, and the three-dimensional analysis the most speculative. In one dimension our arguments make use of a phase that exhibits charge confinement. In higher dimensions, it makes use of superconductivity and the Anderson-Higgs mechanism. Insofar as our analysis relies on a mapping to Ref. [3], it shares similar limitations; viz., we can establish localization only at low (but nonzero) temperatures [54]. Whether infinite-temperature localization can arise with long-range interactions remains an open problem. However, our work opens the door to the study of MBL physics in a host of experimentally relevant low-temperature systems with long-range interactions. We emphasize that our work differs from the classic analysis of Ref. [55] in that it predicts a strictly zero conductivity at nonzero energy density, up to rare region effects. It differs also from Refs. [56,57] in that we do not restrict ourselves to Anderson localization of single spin flips, and consider instead many-body localization.

\section{LOCALIZATION WITH CONFINING INTERACTIONS IN ONE DIMENSION}

We begin with a discussion of one-dimensional systems, where the long-range interaction may be treated exactly using the method of bosonization. We start with a continuum model that is inspired by the Schwinger model [58-61] from high-energy physics. The Schwinger model is a model of quantum electrodynamics in one dimension, which exhibits charge confinement. It is formulated as a one-dimensional Dirac fermion coupled to a gauge field. For our purposes, however, it is more convenient to adopt a description in which the gauge field has been integrated out. The Hamiltonian that we study thus involves Dirac fermions moving in one dimension with a long-range constant force interaction (Coulomb interaction in one spatial dimension). The Hamiltonian is $H_{0}+H_{\text {int }}$, where

$$
\begin{gathered}
H_{0}=\int \frac{d k}{2 \pi} \sum_{r= \pm 1} v\left(r k-k_{F}\right) c_{r, k}^{\dagger} c_{r, k}, \\
H_{\text {int }}=-e^{2} \int d x d y \rho(x)|x-y| \rho(y),
\end{gathered}
$$

and $\rho(x)=\sum_{r= \pm 1} c_{r}^{\dagger}(x) c_{r}(x)$. The argument is cleanest when the theory is formulated in the continuum, in which case an asymptotically large UV cutoff $\Lambda$ must be placed on the dispersion. Lattice formulations of the argument are discussed after we introduce the main argument. The Schwinger model itself has an additional parameter, which is a uniform background electric field (which can be set to have any value). We choose to set this uniform background electric field to zero. Consideration of potential phase transitions driven by uniform background field is deferred to future work. There is a uniform positively charged jellium background (introduced so that the energy density remains finite in the thermodynamic limit). This background is taken to be rigid; i.e., we neglect any coupling to phonons of the jellium.

We now make use of standard bosonization formulas from Ref. [62] to obtain [63]

$$
\begin{gathered}
H_{0}=\int \frac{d x}{(2 \pi)} v_{F}\left\{\pi^{2} \Pi^{2}(x)+[\nabla \phi(x)]^{2}\right\}, \\
H_{\text {int }}=\frac{4 e^{2}}{\pi} \int \frac{d x}{2 \pi} \phi^{2}(x),
\end{gathered}
$$

where $\phi$ describes fluctuations of charge density wave order and we introduce $\Pi$ as the conjugate momentum to the $\phi$ field. We emphasize that for the continuum model Eqs. (1) and (2), the bosonized form Eqs. (3) and (4) is exact. For a lattice regularization of this model the bosonized form will be only asymptotic, with corrections that are irrelevant for the thermodynamics but potentially important for the dynamics. These are discussed in the section on lattice regularizations, Sec. II A. It is convenient, however, to begin with a discussion of the continuum model Eqs. (1) and (2), which exactly bosonizes to Eqs. (3) and (4). This corresponds to a Klein-Gordon theory of free massive scalar bosons. The density pattern in the ground state (at a nonzero fermion density) shows crystalline longrange order [61], and the phase may be thus thought of as a kind of Wigner crystal where the usual constraints on longrange order in one dimension have been evaded because of the long-range interaction. We make this expression look more familiar by casting it into the form

$$
H=\int \frac{d q}{(2 \pi)} u_{q}\left[K_{q} \pi^{2} \Pi(q) \Pi(-q)+\frac{1}{K_{q}} q^{2} \phi(q) \phi(-q)\right],
$$

where

$$
u_{q}=v_{F} \sqrt{1+\frac{2 V_{q}}{\pi v_{F}}}, \quad K_{q}=\frac{1}{\sqrt{1+\frac{2 V_{q}}{\pi v_{F}}}}, \quad V_{q}=\frac{2 e^{2}}{q^{2}} .
$$

Adding short-range interactions will shift $K(q \rightarrow \infty)$ but will not affect the physics of interest to us here. Note that the bosonized description of the Schwinger model involves a noncompact boson-this is a consequence of integrating out the gauge field [64], and will be important to our argument. Note also that so far the transformations performed are exact at the operator level.

We now introduce disorder. We emphasize that the standard prescription in the localization literature of perturbing about the infinite disorder state is unsuitable here because of the long-range interaction. Not only will the 
perturbation theory not converge [52,53], but the infinite disorder state itself is an inappropriate starting point-a region of size $L$ will have charge $\sim \sqrt{L}$ from the central limit theorem, and will thus have an electrostatic energy $\sim L^{3 / 2}$, which will diverge in a superextensive fashion in the limit of large system size. Instead, we first treat the interaction exactly by the method of bosonization, and then introduce disorder. Our analysis is controlled in the regime when disorder is weak compared to the interaction. We emphasize also that the disorder is allowed to backscatter electrons (turn right movers into left movers and vice versa), which is physics that does not typically enter the high-energy literature. The disorder adds to the Hamiltonian a piece $H_{\text {dis }}$, where (see Appendix A)

$H_{\text {dis }}=-\int d x\left[\frac{1}{\pi} \eta(x) \nabla \phi+\left(\frac{\xi^{*}(x)}{2 \pi \alpha} e^{i 2 \phi(x)}+\right.\right.$ H.c. $\left.)\right]$.

Here, $\eta$ represents forward scattering and $\xi$ represents backscattering. We make the standard assumption that $\eta$ and $\xi$ can be modeled as independent short-range correlated Gaussian random variables. Here, $\alpha \sim 1 / \Lambda$ is a UV cutoff.

At this stage, it is tempting to apply the classic Giamarchi-Schulz renormalization group analysis [65], which obtains the $\beta$ functions for the disorder strength $\mathcal{D}$ [defined by $\left\langle\xi^{*}(x) \xi(y)\right\rangle=\mathcal{D} \delta(x-y)$ ], perturbatively in weak disorder. Generalized to the present problem, an analysis of this form gives $[(d \mathcal{D}) / d l]=3 \mathcal{D}$; i.e., disorder is always a relevant perturbation. However, the GiamarchiSchulz calculation is a zero-temperature calculation, whereas we are interested in the behavior at nonzero temperatures. Additionally, we wish to consider an isolated quantum system, disconnected from any external heat bath, such that one cannot use the Matsubara formalism, nor does it make sense to talk about free-energy minimization. Finally, we are interested not in the disorder averaged properties, but rather in the behavior of a single sample in a typical disorder realization.

The analysis of a disordered, interacting Luttinger liquid away from its ground state, without the crutches of disorder averaged field theory or the Matsubara formalism, may appear to be a formidable task $[50,66,67]$. It is a problem, however, that is amenable to analytical treatment. We start by introducing the notation $\xi(x)=D(x) \exp [i \zeta(x)]$, and, hence, rewrite the Hamiltonian (for a particular disorder realization, after an integration by parts) as

$$
\begin{gathered}
H=\int \frac{d x}{2 \pi} v_{F} \pi^{2} \Pi^{2}(x)+v_{F}(\nabla \phi)^{2}+V(\phi), \\
V(\phi)=\frac{4 e^{2}}{\pi} \phi(x)^{2}+\frac{2 D(x)}{\alpha} \cos [2 \phi-\zeta(x)]-2 \tilde{\eta}(x) \phi,
\end{gathered}
$$

where $\tilde{\eta}(x)=\int^{x} d y \eta(y)$, and $\tilde{\eta}, D(x)$, and $\zeta(x)$ are taken to be independent zero-mean random variables with shortrange correlations. We now introduce $\phi_{0}(x)$ to be the static background field configuration that minimizes the Hamiltonian,

$$
v_{F} \partial_{x}^{2} \phi_{0}=\frac{4 e^{2}}{\pi} \phi_{0}(x)-\frac{2 D(x)}{\alpha} \sin \left(2 \phi_{0}-\zeta\right)-\tilde{\eta} .
$$

Note that $\phi_{0}$ simply represents the adjustment of the Wigner crystal to the disorder that we introduce; i.e., it represents the classical ground state, where all quantum fluctuations have been ignored. We assume that $D(x)$ is a bounded random variable $0<D(x)<D_{0}$, with $D_{0} / \alpha<e^{2} / \pi$, so that $V(\phi)$ has a unique minimum. We reintroduce quantum fluctuations by writing $\phi(x)=\phi_{0}+\delta \phi$, and obtain the effective Hamiltonian as a power series in small $\delta \phi$. This expansion is well behaved since $V(\phi)$ has a unique minimum, in contrast to the situation that is obtained for compact potentials [62], where instantons connecting distinct minima must be taken into account. Relabeling $\delta \phi$ simply as $\phi$, we obtain the Hamiltonian (up to an unimportant additive constant) as

$$
\begin{aligned}
H= & \int \frac{d x}{2 \pi} v_{F} \pi^{2} \Pi^{2}(x)+v_{F}(\nabla \phi)^{2} \\
& +\left[\frac{4 e^{2}}{\pi}-\frac{4 D(x)}{\alpha} \cos \left(2 \phi_{0}-\zeta\right)\right] \phi^{2}+O\left(\phi^{3}\right) .
\end{aligned}
$$

At leading order this is simply a theory of noninteracting gapped bosons in a random potential, which is well known to have all its (single-particle) eigenstates localized (see, e.g., Ref. [68] for an explicit discussion). The higher-order terms come from the expansion of the cosine, are short ranged in real space, and may be treated within a perturbative locator expansion. Perturbative locator expansions of this form were shown to converge at sufficiently low (but nonzero) energy densities [3] (up to possible rare region corrections [47]), and thus localization should persist even when nonlinearities from higher-order terms in the expansion are taken into account; i.e., the Hamiltonian Eq. (8) should exhibit manybody localization at sufficiently low (but nonzero) energy densities.

There is a subtlety to be noted here. Given that we are working in the continuum, the single-particle localization length is unbounded above, whereas Ref. [3] assumes a bounded localization length. Problems with unbounded single-particle localization length and shortrange interactions have been studied in the MBL literature $[42,69,70]$. Reference [42] showed that as long as $\Upsilon=$ $g^{\xi^{3 d}}(E) P(E)^{2}[1-P(E)]^{2}$ is small everywhere in the spectrum [where $g=D / \alpha$ is the nonlinearity strength, $\xi(E)$ is the localization length, and $P(E)$ is the occupation number for a system prepared in a Gibbs state parametrized by a temperature $T$ ], the locator expansion converges at a typical point in space. Now, at small energy densities (i.e., low temperatures), and at small $E$, we surely have $\Upsilon \ll 1$. Meanwhile, since $\xi(E)$ and $g(E)$ both grow as power-law 
functions of energy, but the $P(E)$ function decays as an exponential function of energy, we continue to have $\Upsilon \ll 1$ at high energies, and a mapping onto Ref. [3] to establish perturbative stability of MBL at a typical point in space is possible [69].

Reference [42] also raised the possibility that rare "hot" regions may break the locator expansion in the continuum. This scenario was recently studied in detail in Ref. [70], where it was concluded that rare regions would always lead to delocalization, with a relaxation time scale that diverged faster than $\exp (1 / T)$ at low temperatures. This "rare region" problem is endemic to models with many-body mobility edges [47], and the present model is no exception. Whether the rare region problem can be circumvented remains an open problem, which, however, has nothing to do with the long-range interacting nature of the problemthe Hamiltonian Eq. (8) is just as localized as would be a short-range interacting problem in the continuum. Indeed, the charge confinement in the model makes the interactions between the available degrees of freedom effectively short range (recall that interactions between dipoles in one dimension are not long range), and thus many-body localizable in the usual manner, even though the underlying Hamiltonian had long-range interactions.

We now estimate the localization length for the low-lying excited states. This is approximately the single-particle localization length (since perturbation theory in the interaction converges at typical points in space). In one dimension this is proportional to the scattering length $l$, which can be calculated in a self-consistent Born approximation (SCBA) with the Green function $G=\left[1 /\left(E-4 e^{2} / \pi-\hbar v_{F} k^{2} \alpha\right)\right]$, cutoff on the length scale $l$. For states just above the gap this gives $l=\left(\hbar v_{F} / D_{0} \Xi\right)^{2 / 3} \alpha$, where $D_{0} \Xi$ is the Fourier transform of the disorder potential (i.e., $\Xi$ is a disorder correlation length) and $\alpha$ is the UV cutoff length scale. The analysis is controlled only when $D_{0} \Xi \ll \hbar v_{F}$; i.e., disorder is weak compared to the kinetic energy scale.

This localization length scale should be compared to the de Broglie length $\lambda$, set by the inhomogeneity of the potential. If the disorder is weak (as we are assuming), then the de Broglie wavelength will be long, and so we should account for central limit averaging of the disorder over one de Broglie wavelength. We then have to solve

$\frac{\hbar v_{F}}{\lambda^{2}}=\frac{D_{0}}{\alpha} \sqrt{\Xi / \lambda} \Rightarrow \lambda=\left(\hbar v_{F} / D_{0} \Xi\right)^{2 / 3}\left(\alpha^{2} \Xi\right)^{1 / 3}$.

Note that self-consistency requires $\lambda \gg \Xi$, which is automatically ensured at weak disorder. We can now observe that $(l / \lambda)=(\alpha / \Xi)^{1 / 3}$ is the standard control parameter for weak localization theory [71]. When $\Xi>\alpha$ (such that the UV cutoff is the smallest length scale in the problem), then $l / \lambda \ll 1$; i.e., the lowest-lying excited states are deep in the locator limit.

\section{A. Lattice regularizations}

Thus far, we have worked with a model in the continuum. We now discuss lattice regularizations. The natural tight-binding lattice generalization of the continuum problem we discuss above is

$H=\sum_{k}[E(k)-\mu] c_{k}^{\dagger} c_{k}-e^{2} \sum_{x y} \rho_{x} \rho_{y}|x-y|+\sum_{x} \mu_{x} \rho_{x}$,

where $\mu_{x}$ is a random potential, and where $E(k)$ is the band structure of the lattice Hamiltonian. We specialize to fermions at incommensurate filling, leaving the problem of commensurate fillings to future work. Standard phenomenological bosonization $[62,72,73]$ then predicts that the bosonized Hamiltonian will take the form $H_{l}+H_{\mathrm{nl}}$, where $H_{l}$ is Eq. (8) with integrals replaced by sums and continuum derivatives replaced by lattice derivatives, and $H_{\mathrm{nl}}$ contains nonlinear corrections [terms of the form $(\nabla \phi)^{3},(\nabla \phi)^{4}$, etc.] coming from band curvature. At incommensurate filling, when the density can be replaced by the smeared density, the interaction bosonizes to a sum of local terms, and while bosonization does produce nonlinear terms, these are strictly short range [72,73]. Thus, the problem still maps (after manipulations analogous to those discussed above in the continuum) to a problem of massive bosons in a random potential with short-range interactions. One can again appeal to Ref. [3] to argue that this problem should be many-body localized.

We note that by going to a lattice we eliminate the problem of an unbounded-above single-particle localization length that complicates the analysis in the continuum. However, since the bosonization formulas are applicable only for "almost linear" dispersions, our analysis is still restricted to low (but nonzero) energy densities, when linearization about a Fermi surface is a sensible starting point [74]. Whether infinite-temperature MBL can arise here is an open problem that we leave to future work. Parenthetically, we note that a recent numerical study [75] does observe signatures of MBL in the dynamics of the Schwinger model, but operates in a regime very different from ours, where the interaction energy scale is the largest energy scale in the problem.

\section{LOCALIZATION WITH COULOMB INTERACTIONS IN HIGHER DIMENSIONS}

We now discuss how low-temperature MBL may arise in higher-dimensional systems with long-range interactions. The most natural generalization of our one-dimensional example would involve considering Wigner crystals in higher dimensions. However, the distortions of the Wigner crystal interact via dipolar interactions [76], which in dimensions higher than one are not purely short range. It turns out that if the interaction is sufficiently long range to 
prohibit dissociation of dipoles, then the interaction between dipoles is itself sufficiently long range to obstruct a locator expansion [52]. Conversely, if the interaction between dipoles is sufficiently short range to allow for a locator expansion, then the energy cost of dissociating a dipole is finite, such that at nonzero energy density there exists a nonzero density of free charges, which interact via the "bare" long-range interaction. Thus, the obvious generalization of our discussion to higher dimensions is problematic.

A more fruitful line of attack is opened up by viewing our one-dimensional problem as an example of a confining phase [58,59]. Given the intimate connections between confinement and the Anderson-Higgs mechanism [77], we are therefore prompted to consider Higgsed phases (e.g., superconductors) as a possible platform for higherdimensional MBL with long-range interactions. We therefore focus in this section on using superconductivity to eliminate the long-range charge interaction, and to obtain a description of a correlated phase in terms of emergent excitations with purely short-range interactions, which may then be many-body localized. We begin with a discussion in two dimensions, before generalizing to threedimensional systems. The argument works equally well in the continuum or on the lattice, modulo the usual subtleties with localization in the continuum $[42,69,70]$. A jellium background is again assumed, so that the uniform state has finite electrostatic energy in the thermodynamic limit.

It is imperative that we do not have phonons in the problem, since phonons (and Goldstone modes in general) have a diverging single-particle localization length at low energies [78], which is believed to pose an obstruction to MBL $[41,79]$. We thus need a purely electronic mechanism for superconductivity. We use the Kohn-Luttinger theorem to this end $[80,81]$ as a key building block for our analysis. The Kohn-Luttinger argument in the continuum [80,81] shows that a long-range isotropic repulsion generates through perturbation theory a short-range attraction in a sufficiently high angular momentum channel, which can induce superconductivity. Lattice versions of the argument are also known (see, e.g., Refs. [82,83] for recent discussions). That superconductivity arises in a high angular momentum channel is a feature, since these superconductors lack the protection against disorder that $s$-wave superconductors inherit from the Anderson theorem [84], and are thus easier to localize. However, it is important that the superconductivity should be nonchiral, since chiral states possess their own obstructions to localization [41]. This may be accomplished either by working on a lattice where the Kohn-Luttinger attraction arises in a one-dimensional irreducible representation of the lattice symmetry group (see, e.g., Ref. [83] for a specific example) or in the continuum, if the energetics favor a nodal rather than a chiral state. It is also imperative that there should not be a spin $\mathrm{SU}(2)$ symmetry in the problem, since $\mathrm{SU}(2)$ symmetry poses its own obstruction to MBL [45,85]. This may be evaded either by working with spinless fermions or by applying a Zeeman field to break the spin symmetry down to $\mathrm{U}(1)$.

We now discuss how superconductivity enables MBL in a long-range interacting system. The argument is independent of the precise mechanism of superconductivity (as long as it is not mediated by the Goldstone bosons of some continuous symmetry, e.g., acoustic phonons) and also of the particular structure of the superconducting ground state - as long as it is nonchiral, not protected by the Anderson theorem, and has low enough symmetry that there are no higher-dimensional irreducible representations [45]. We emphasize that we are discussing here not superfluidity, but rather true superconductivity, i.e., the charges are coupled to a dynamical gauge field, and the Goldstone mode is gapped out by the Anderson-Higgs mechanism. We emphasize also that we are discussing a superconductor treated as a closed quantum system, which is not in thermodynamic equilibrium. Additionally, the superconductor is disordered, but the disorder is not so strong as to destroy superconductivity.

Once the system becomes superconducting, the longrange interaction is screened out. The effective degrees of freedom in a superconductor are the Bogolioubov-de Gennes quasiparticles, the vortices, and bound states of the two [86,87], as well as the photons which mediate the electromagnetic interaction. We emphasize that the correctly formulated excitations carry neither charge nor dipole moment on long length scales [88]. This must be the case, since otherwise there would be electromagnetic fields at long length scales, which is inconsistent with Meissner physics. For an $s$-wave superconductor in two dimensions, the effective theory for quasiparticles and vortices is simply the toric code [86,87], the topologically ordered phase of which is the superconductor. The disordered toric code has been shown [27] to support topological order even in its excited states, from which it follows that an isolated two-dimensional $s$-wave superconductor can exhibit superconductivity even away from its ground state, with vortices and quasiparticles localized on disorder. The present problem differs somewhat in that the quasiparticles are nodal rather than gapped. However, at the level of the noninteracting theory, it is known that a twodimensional disordered nodal superconductor supports an Anderson localized phase for the quasiparticles [89-91]. Meanwhile, the interactions (between vortices, between quasiparticles, and between quasiparticles and vortices) have been derived in, e.g., Ref. [91], and are purely short ranged. If we can also demonstrate localization of the photon mode, it will then follow from Ref. [3] that a system of localized quasiparticles and vortices with weak shortrange interactions will be in a many-body localized phase, notwithstanding that the bare electronic Hamiltonian contained a long-range interaction. 
We now discuss the localization of the photon mode. In the superconductor the photon mode is gapped out by the Higgs mechanism, and can thus be ignored when groundstate physics is the main concern, as in Refs. [89-91]. However, since we aim to establish MBL at low but nonzero temperatures, the photon mode must be taken into account. We now offer two arguments that the photon mode is also localized, and thus does not materially alter the conclusions reached above. Both arguments are adapted from the equivalent arguments for Goldstone modes in Ref. [78]. Note that in the case of the superconductor, the Goldstone mode does not exist as a separate excitation, but instead is absorbed into the photon mode via the AndersonHiggs mechanism.

We wish to describe a superconductor with order parameter $\Delta(r) \exp [i \theta(r)]$ minimally coupled to a gauge field $\left(A_{0}, \mathbf{A}\right)$ which lives in two dimensions. The effective theory for this is the Abelian Higgs model [86,87], for which the equation of motion takes the form [92]

$$
\left[\partial_{t}^{2}-c_{L, T} \nabla^{2}+\Delta(r)^{2}\right] \mathbf{A}_{L, T}=0,
$$

where $\Delta(r)$ is the (spatially inhomogeneous) gap function, $A_{L}\left(A_{T}\right)$ is the longitudinal (transverse) photon mode (the longitudinal photon mode being the remnant of the plasma oscillation mode in the normal metal), and $c_{L}\left(c_{T}\right)$ is the longitudinal (transverse) photon velocity. In a physical superconductor, $c_{T}$ is the speed of light while $c_{L}$ is of order Fermi velocity. If the speed of light is taken to infinity (so that the interaction is instantaneous), then the transverse mode can be neglected as infinitely energetic, but the longitudinal polarization must still be taken into account. Note that disorder enters through a mass term, i.e., the disorder vertex does not vanish at low frequency, and the dispersion relation at low frequency takes the form $\omega^{2} \approx \Delta(r)^{2}+q^{2} \Rightarrow \omega=\Delta+q^{2} / 2 \Delta$. We now follow Ref. [78] and first estimate a mean free path $l$ from SCBA, and then substitute $k l$ into weak localization theory, where $k$ is the clean system wave vector corresponding to a frequency $\omega$. This analysis reveals that in spatial dimensions $d=1,2,3, k l$ is free of divergences at low frequency, such that all low-energy photon modes can be localized with bounded localization length, in sharp contrast to (nonHiggsed) Goldstone modes [78] (see Appendix B for explicit calculation). Moreover, the interactions between photon modes and order parameter fluctuations are strictly short range, so the photon sector does not present any obstruction to localization. Since all modes involving spatially nonuniform rotations of the phase are localized with bounded localization length at low energy, the obstructions to MBL discussed in Refs. [41,79] do not apply. There is of course still the global mode involving spatially uniform rotations of phase. It is not clear to us what it would mean for such a mode to be localized or extended. In any case, such a global mode cannot be used to form spatially localized wave packets or to transport energy, so its fate is irrelevant to our present discussion.

An alternative argument, also adapted from Ref. [78], proceeds as follows. The dispersion relation for the plasmon mode takes the form

$\omega^{2}=\Delta^{2}+q^{2}+2 \Delta m(r) \Rightarrow 2 \Delta \tilde{\omega} \sim q^{2}+\Delta m(r)$,

where $m$ is the (zero-mean) fluctuation in the gap function and $\Delta$ is the mean gap function, and to obtain the second expression we take the scaling limit $\omega \rightarrow \Delta$ and define $\tilde{\omega}=\omega-\Delta$. Now, performing central limit averaging on the disorder over one wavelength of the clean system, we obtain

$$
2 \Delta \tilde{\omega} \sim q^{2}+\Delta m_{0} q^{d / 2}
$$

where $m_{0}$ is the typical fluctuation in the gap function. For $d<4$, the disorder term dominates the low-energy dispersion relation (i.e., disorder is relevant). One can estimate a localization length $\xi$ in the scaling limit by setting $\tilde{\omega} \sim q^{2}$ and $q \approx \xi^{-1}$ and solving to obtain $\xi \sim m_{0}^{-2 /(4-d)}$, which is finite for $d=1,2,3$ and in $d=1$ agrees with our earlier results, identifying $m_{0} \leftrightarrow D_{0} \Xi$.

It thus follows that the isolated disordered twodimensional superconductor is described by an effective theory in which all sectors are localized with bounded single-particle localization length at low energies, and with short-range interactions. It then follows from Ref. [3] that a many-body localized phase should exist, at least at lowenergy densities, notwithstanding that the bare Hamiltonian contained long-range interactions. Of course, our entire discussion is valid only in the low-energy part of the spectrum, at energy densities below the gap scale. As such, the rare region scenario endemic to problems with manybody mobility edges arises here also. Whether infinitetemperature MBL can be obtained (or the rare region problems circumvented in some other way) is a problem that we leave to future work.

We now offer an alternative, intuitive way to understand our results. A Hamiltonian with a Gauss law interaction (like $\ln r$ in two spatial dimensions) can always be rewritten as a purely local Hamiltonian, with only short-range interactions, by introducing a gauge field. Absent superconductivity, the obstruction to construction of a locator expansion enters in this representation through the back door, because the gauge field itself possesses an obstruction to localization [78], and a system where one of the sectors is protected against localization does not admit of a locator expansion [41,48,50,79]. However, once we Higgs the gauge field it loses its protection against localization, and a local Hamiltonian where none of the sectors is protected against localization can be many-body localized in the usual manner. 
We speculate that our arguments for MBL with longrange interactions may also extend to three-dimensional systems with $1 / r$ interactions. The basic argument follows analogously to two dimensions (the most trivial extension involves Josephson coupled superconducting layers). The gauge field now lives in three spatial dimensions, but it follows from our discussion of photon localization above that the gauge field is localized with bounded localization length at low frequency, even in $d=3$. However, there are some differences in three spatial dimensions, the full implications of which remain to be understood. One significant difference is that vortices in a three-dimensional superconductor are linelike objects, and one cannot argue for their localization based simply on appeals to Ref. [3], which discusses localization of pointlike excitations. This problem may be circumvented in one of two ways. Either one can work in the sector with no vortex excitations (easier to accomplish in three dimensions since vortex-antivortex pairs cost an energy that diverges linearly with the length of the vortex line) or, alternatively, one can appeal to the body of work establishing the existence of a glassy phase of vortices that persists up to nonzero temperature in thermodynamic equilibrium $[93,94]$. If a vortex glass phase exists at finite temperature in thermodynamic equilibrium, then it seems plausible that a localized phase of vortices should also exist at finite energy density in an isolated quantum system.

Another point to note is that in three dimensions, the problems associated with being in the continuum (even with short-range interactions) are much more severe, since delocalized single-particle states arise above a critical energy. These delocalized single-particle states are difficult to reconcile with MBL (however, see Refs. [95-98]). The canonical way to regulate problems arising at high energies in the continuum is to place the theory on a lattice. In a lattice gauge theory, where the photons are also placed on a lattice, one has simply a theory of $Z_{2}$ topological order [86], which can be many-body localized [27] in the usual manner. However, for physical superconductors, the electrons live on a lattice, but the gauge field lives in the continuum. As such, delocalized photon modes unavoidably appear at high energies. However, high-energy photons are also noninteracting, because Maxwell's equations in vacuum are linear. Whether such noninteracting but delocalized high-energy photon modes endow the system with a finite relaxation time, and how long the relaxation time is if so, is a subtlety that remains to be understood. A detailed investigation of these issues is left to future work.

\section{DISCUSSION}

We plausibly argue that systems with long-range interactions can be in a many-body localized phase. The basic idea is that the long-range interaction can drive the system into a correlated phase naturally described in terms of emergent excitations with only short-range interactions, which can be many-body localized in the usual manner. We demonstrate this for a one-dimensional problem of fermions with $\sim r$ interactions, and for a two-dimensional problem of fermions with $\log r$ repulsion, and speculate that similar arguments may also apply to fermions in three dimensions with $\sim 1 / r$ repulsion; i.e., many-body localization is compatible with Coulomb repulsion in all physical dimensions. Our arguments lean on Ref. [3] and are thus asymptotic in the sense that they only establish localization at typical points in space. Truly rigorous proofs of MBL, such as Ref. [7], require a consideration also of rare region effects, which may render conductivity finite even when typical regions appear localized. Such effects are known to be particularly severe in dimensions greater than one [49]. With that said, rare region obstructions have little to do with whether the interactions are long range or not, and, moreover, most existing rare region obstructions to MBL assume short-range correlated disorder, and may not generalize to situations where the disorder is, e.g., quasiperiodic. A fully rigorous treatment including rare region effects would be an interesting direction for future work, but is beyond the scope of the present paper.

Our work brings into focus a host of additional conceptual questions. For example, does low-temperature MBL have a description in terms of emergent local integrals of motion, similar to infinite-temperature MBL? Recent work [18] has provided the beginnings of an answer, but much remains to be understood. Also open is the question of whether MBL can arise in mixed dimensional problems, e.g., systems of fermions moving in two dimensions, but interacting via a $1 / r$ potential mediated by a gauge field that lives in three dimensions. Prima facie this seems unlikely, since disorder in two dimensions will not localize a gauge field that lives in three dimensions, and the three-dimensional gauge field could then act as a higher-dimensional bath to delocalize the system [48]; however, the problem deserves more careful consideration.

A particularly interesting open question is whether MBL can arise for interactions of range intermediate between Coulomb and short range, e.g., the experimentally relevant case of dipolar interactions [38]. On physical grounds, one could argue that if Coulomb interactions admit of MBL, and short-range interactions admit of MBL, then interactions of intermediate range should admit of MBL also. However, the particular methods we employ to establish MBL with Coulomb interactions do not readily generalize to interactions of intermediate range. In one dimension, a density-density interaction bosonizes to $V(q) q^{2} \phi^{2}$, where $V(q)$ is the Fourier transform of the potential. The confining potential $V(r) \sim-r$ is special in that it has $V(q) \sim 1 / q^{2}$, which produces a mass gap in the bosonic spectrum. A less long-range interaction would bosonize to a term of the form $q^{\alpha} \phi^{2}$, where $0<\alpha<2$, and this would 
not open up a mass gap. The mass gap, we remind the reader, is important to our argument in that it produces a noncompact potential with a unique minimum, allowing us to ignore instanton events. A term like $q^{\alpha} \phi^{2}$ with $\alpha>0$ would leave us with a problem of bosons with a complicated dispersion in a compact potential, which does not appear amenable to analytical solution. Similarly, two-dimensional problems with $\log r$ interactions, and three-dimensional problems with $1 / r$ interactions, are also special in that this interaction can be mediated by a gauge field with a natural kinetic energy, allowing us to map the long-range interacting problem to a local theory (the Abelian Higgs model) in which all sectors can be localized. Alternative power laws for the interaction will not exhibit this nice property. Thus, while physically it seems plausible that interactions of intermediate range should also admit of localization, the particular methods we employ herein do not readily generalize, and a demonstration of MBL in such systems will require fresh ideas. One possibility may be to use the generalized Gauss laws that arise for higher rank gauge fields [99]. A detailed investigation of the possibility of MBL with intermediate-range interactions is left to future work.

Finally, it is interesting to ask what other types of correlated phase could serve as stepping stones to MBL physics in long-range interacting systems, besides the confined and Higgsed phases we discuss herein. Notwithstanding these open questions, however, our demonstration of low (but nonzero) temperature MBL in long-range interacting systems already opens the door to investigation of MBL physics in a host of experimentally relevant systems with long-range interactions.

\section{ACKNOWLEDGMENTS}

We acknowledge useful conversations with Victor Gurarie and Ana Maria Rey. We thank Sarang Gopalakrishnan for feedback on the manuscript. We also acknowledge Ahmed Akhtar and M. C. Banuls for an ongoing collaboration on related ideas. This material is based in part upon work supported by the Air Force Office of Scientific Research under Award No. FA9550-17-10183 (R. M. N.). R. M. N. also acknowledges the support of the Sloan Foundation through a Sloan Research Fellowship. S. L.S. is supported in part by the U.S. Department of Energy under Grant No. DE-SC0016244.

\section{APPENDIX A: BOSONIZATION}

In this appendix, we provide more details regarding the bosonization procedure employed in the main text. We begin with a fermionic Hamilltonian of the form $H=H_{0}+H_{\text {int }}+H_{\text {dis }}$, where $H_{0}$ is given by Eq. (1), $H_{\text {int }}$ by Eq. (1), and where
$H_{\mathrm{dis}}=\int d x V(x) c^{\dagger}(x) c(x)$, where $c=c_{1}+c_{-1}$; thus,
$H_{\mathrm{dis}}=\int d x V(x)\left[\sum_{r= \pm 1} c_{r}^{\dagger}(x) c_{r}(x)+\left[c_{1}^{\dagger}(x) c_{-1}(x)+\right.\right.$ H.c. $\left.]\right]$.

Here, $V(x)$ is a spatially random chemical potential. Note that $V(x)$ can scatter between right- and left-moving states. We take $V(x)$ to be short-range correlated in space, with $\langle V(x)\rangle=0$ and $\left\langle V(x) V\left(x^{\prime}\right)\right\rangle=\mathcal{D} K\left(x-x^{\prime}\right)$, where $K\left(x-x^{\prime}\right)$ integrates to unity and is only nonzero over some finite range.

We now make use of the bosonization transformations detailed in Appendix D of Ref. [62], and conclude that $H_{0}$ bosonizes to the form Eq. (3), $H_{\text {int }}$ bosonizes to the form Eq. (4), and $H_{\text {dis }}$ bosonizes to the form Eq. (7), with the identification $\eta(x)=V(x)$ and $\xi(x)=V(x) e^{-2 i k_{F} x}$. It follows that $\eta$ and $\xi$ are both zero-mean random variables, whose correlation length is no longer than the correlation length for $V(x)$. The rest of our discussion follows as in the main text.

We emphasize that the bosonization transformations leading to Eqs. (3), (4), and (7) are exact up to corrections that are suppressed by powers of the UV cutoff. Thus, the bosonization transformations are exact as long as the interaction and disorder energy scales are much smaller than the bandwidth of $H_{0}$. The exactness of the bosonization transformations is a peculiarity of the continuum model with perfect linear dispersion. In a lattice model, additional terms will be generated, complicating the analysis.

We note also that while the bosonization transformations do not require disorder to be weaker than the interaction, it is nevertheless convenient for our analysis for the scales to be ordered so. This is because taking the disorder to be weaker than the interaction ensures diluteness of domain walls in the ground state, which stabilizes the locator expansion. If the disorder energy scale is large compared to the gap (set by the interaction), then domain walls are dense even in the ground state, and our analysis is ill controlled.

\section{APPENDIX B: LOCALIZATION OF HIGGSED PLASMON}

In this appendix, we generalize the calculation of Ref. [78] to a Higgsed Goldstone mode, and demonstrate that the localization length is bounded at low energies in spatial dimensions $d=1,2,3$.

Let us first review the calculation of Ref. [78], which considers phonons (Goldstone modes) in a random medium, with the equation of motion

$$
\omega^{2} \phi(r)=c(r)^{2} \nabla^{2} \phi(r) .
$$

Note that the disorder enters through a term that also carries spatial derivatives. Meanwhile, the Green functions of the 
phonon field take the form $G(\omega, k)=\left[1 /\left(\omega-E_{k}-i / \tau\right)\right]$, where the scattering time $\tau$ comes from scattering off disorder and $E_{k}=c k$, with $c$ being the mean phonon speed. The scattering time may be estimated from the selfconsistent Born Approximation (SCBA), whereupon one has to solve the self-consistent equation,

$$
1=g(\omega) \int \frac{k^{d-1} d k}{\left(\omega-E_{k}\right)^{2}+1 / \tau^{2}} .
$$

Here, $\omega$ is the phonon frequency and the disorder strength $g(\omega) \sim \omega^{2}$, because the disorder enters in a term that involves spatial derivatives, such that the coupling to disorder vanishes at long wavelengths or low frequencies. For $E_{k} \sim k$ this yields $\tau^{-1} \sim \omega^{d+1}$, and a mean free path $l \sim \tau \sim \omega^{-(d+1)}$. In one dimension, the localization length is proportional to the mean free path, so $\xi_{1 \mathrm{D}} \sim \omega^{-2}$ diverges as a power law at low frequency. In two dimensions, weak localization theory predicts $\xi_{2 \mathrm{D}}=\exp (k l)$. Taking $k=\omega$ and $l=\omega^{-3}$, we obtain $\xi_{2 \mathrm{D}}=\exp \left(1 / \omega^{2}\right)$, which diverges exponentially fast at low frequencies. In three dimensions, we have $l \sim \omega^{-4}$ and $k l \sim \omega^{-3}$. In three dimensions weak localization theory predicts a delocalized phase for large $\mathrm{kl}$; i.e., the low-frequency phonon states are delocalized.

The analysis can be readily generalized to a Higgsed plasmon mode, to determine whether the localization length diverges close to the gap edge. Once again, self-consistent Born approximation yields an expression of the form Eq. (B2). However, since the disorder in Eq. (14) enters through a term that is independent of spatial derivatives, the disorder vertex is frequency independent at low frequency, $g(\omega) \sim g$. Additionally, the dispersion is modified to $E_{k} \sim$ $\Delta+k^{2}$ [Eq. (15)]. SCBA now predicts $\tau^{-1} \sim(\omega-\Delta)^{(d-1) / 3}$ and a mean free path $l \sim \tau^{1 / 2} \sim(\omega-\Delta)^{(1-d) / 6}$. In one dimension, the localization length is proportional to the mean free path, which remains finite as $\omega \rightarrow \Delta$. In two and three dimensions, the control parameter for weak localization theory, $k l \sim(\omega-\Delta)^{(4-d) / 6}$, is divergence free at low frequency, and thus all states remain localized, even arbitrarily close to the gap edge. Indeed, $k l$ vanishes close to the gap edge, indicating that close to the gap edge states are in the Ioffe-Regel strong scattering regime where weak localization gives way to strong localization [1]. Additionally, in the presence of disorder there will be Lifshitz tail states in the gap $\omega<\Delta$, but these are expected to be localized with bounded localization length in any dimension. One thus concludes that a Higgsed plasmon mode can have all its low-frequency states localized with bounded localization length. This conclusion is also consistent with the alternative argument adapted from Ref. [78], presented in the main text.

[1] P. W. Anderson, Absence of Diffusion in Certain Random Lattices, Phys. Rev. 109, 1492 (1958).
[2] I. V. Gornyi, A. D. Mirlin, and D. G. Polyakov, Interacting Electrons in Disordered Wires: Anderson Localization and Low-T Transport, Phys. Rev. Lett. 95, 206603 (2005).

[3] D. M. Basko, I. L. Aleiner, and B. L. Altshuler, MetalInsulator Transition in a Weakly Interacting ManyElectron System with Localized Single-Particle States, Ann. Phys. (Amsterdam) 321, 1126 (2006).

[4] M. Žnidarič, T. ž Prosen, and P. Prelovšek, Many-Body Localization in the Heisenberg XXZ Magnet in a Random Field, Phys. Rev. B 77, 064426 (2008).

[5] V. Oganesyan and D. A. Huse, Localization of Interacting Fermions at High Temperature, Phys. Rev. B 75, 155111 (2007).

[6] A. Pal and D. A. Huse, Many-Body Localization Phase Transition, Phys. Rev. B 82, 174411 (2010).

[7] J. Z. Imbrie, Diagonalization and Many-Body Localization for a Disordered Quantum Spin Chain, Phys. Rev. Lett. 117, 027201 (2016).

[8] R. Nandkishore and D. A. Huse, Many-Body Localization and Thermalization in Quantum Statistical Mechanics, Annu. Rev. Condens. Matter Phys. 6, 15 (2015).

[9] S. S. Kondov, W. R. McGehee, W. Xu, and B. DeMarco, Disorder-Induced Localization in a Strongly Correlated Atomic Hubbard Gas, Phys. Rev. Lett. 114, 083002 (2015).

[10] M. Schreiber, S. S. Hodgman, P. Bordia, H. P. Luschen, M. H. Fischer, R. Vosk, E. Altman, U. Schneider, and I. Bloch, Observation of Many-Body Localization of Interacting Fermions in a Quasirandom Optical Lattice, Science 349, 842 (2015).

[11] J. Smith, A. Lee, P. Richerme, B. Neyenhuis, P. W. Hess, P. Hauke, M. Heyl, D. A. Huse, and C. Monroe, Many-Body Localization in a Quantum Simulator with Programmable Random Disorder, Nat. Phys. 12, 907 (2016).

[12] J. y. Choi, S. Hild, J. Zeiher, P. Schauss, A. Rubio-Abadal, T. Yefsah, V. Khemani, D. A. Huse, I. Bloch, and C. Gross, Exploring the Many-Body Localization Transition in Two Dimensions, Science 352, 1547 (2016).

[13] J. H. Bardarson, F. Pollmann, and J. E. Moore, Unbounded Growth of Entanglement in Models of Many-Body Localization, Phys. Rev. Lett. 109, 017202 (2012).

[14] M. Serbyn, Z. Papić, and D. A. Abanin, Local Conservation Laws and the Structure of the Many-Body Localized States, Phys. Rev. Lett. 111, 127201 (2013).

[15] D. A. Huse, R. Nandkishore, and V. Oganesyan, Phenomenology of Fully Many-Body-Localized Systems, Phys. Rev. B 90, 174202 (2014).

[16] V. Ros, M. Müller, and A. Scardicchio, Integrals of Motion in the Many-Body Localized Phase, Nucl. Phys. B891, 420 (2015).

[17] A. Chandran, A. Pal, C. R. Laumann, and A. Scardicchio, Many-body localization beyond eigenstates in all dimensions, Phys. Rev. B 94, 144203 (2016).

[18] S. D. Geraedts, R. N. Bhatt, and R. Nandkishore, Emergent Local Integrals of Motion without a Complete Set of Localized Eigenstates, Phys. Rev. B 95, 064204 (2017).

[19] S. A. Parameswaran and S. Gopalakrishnan, Non-Fermi Glasses: Localized Descendants of Fractionalized Metals, Phys. Rev. Lett. 119, 146601 (2017). 
[20] V. Khemani, R. Nandkishore, and S. L. Sondhi, Nonlocal Adiabatic Response of a Localized System to Local Manipulations, Nat. Phys. 11, 560 (2015).

[21] S. Gopalakrishnan, M. Müller, V. Khemani, M. Knap, E. Demler, and D. A. Huse, Low-Frequency Conductivity in Many-Body Localized Systems, Phys. Rev. B 92, 104202 (2015).

[22] J. H. Bardarson, F. Pollmann, and J. E. Moore, Unbounded Growth of Entanglement in Models of Many-Body Localization, Phys. Rev. Lett. 109, 017202 (2012).

[23] S. D. Geraedts, R. Nandkishore, and N. Regnault, ManyBody Localization and Thermalization: Insights from the Entanglement Spectrum, Phys. Rev. B 93, 174202 (2016).

[24] V. Khemani, S. P. Lim, D. N. Sheng, and D. A. Huse, Critical Properties of the Many-Body Localization Transition, Phys. Rev. X 7, 021013 (2017).

[25] Z.-C. Yang, A. Hamma, S. M. Giampaolo, E. R. Mucciolo, and C. Chamon, Entanglement Complexity in Quantum Many-Body Dynamics, Thermalization and Localization, Phys. Rev. B 96, 020408 (2017).

[26] S. D. Geraedts, N. Regnault, and R. M. Nandkishore, Characterizing the Many-Body Localization Transition through the Entanglement Spectrum, arXiv:1705.00631.

[27] D. A. Huse, R. Nandkishore, V. Oganesyan, A. Pal, and S. L. Sondhi, Localization-Protected Quantum Order, Phys. Rev. B 88, 014206 (2013).

[28] D. Pekker, G. Refael, E. Altman, E. Demler, and V. Oganesyan, Hilbert-Glass Transition: New Universality of Temperature-Tuned Many-Body Dynamical Quantum Criticality, Phys. Rev. X 4, 011052 (2014).

[29] R. Vosk and E. Altman, Dynamical Quantum Phase Transitions in Random Spin Chains, Phys. Rev. Lett. 112, 217204 (2014).

[30] L. D'Alessio and A. Polkovnikov, Many-Body Energy Localization Transition in Periodically Driven Systems, Ann. Phys. (Amsterdam) 333, 19 (2013).

[31] A. Lazarides, A. Das, and R. Moessner, Fate of Many-Body Localization under Periodic Driving, Phys. Rev. Lett. 115, 030402 (2015).

[32] P. Ponte, A. Chandran, Z. Papić, and D. A. Abanin, Periodically Driven Ergodic and Many-Body Localized Quantum Systems, Ann. Phys. (Amsterdam) 353, 196 (2015).

[33] L. D'Alessio and M. Rigol, Long-Time Behavior of Isolated Periodically Driven Interacting Lattice Systems, Phys. Rev. X 4, 041048 (2014).

[34] H. Kim, T. N. Ikeda, and D. A. Huse, Testing Whether All Eigenstates Obey the Eigenstate Thermalization Hypothesis, Phys. Rev. E 90, 052105 (2014).

[35] V. Khemani, A. Lazarides, R. Moessner, and S. L. Sondhi, Phase Structure of Driven Quantum Systems, Phys. Rev. Lett. 116, 250401 (2016).

[36] D. V. Else, B. Bauer, and C. Nayak, Floquet Time Crystals, Phys. Rev. Lett. 117, 090402 (2016).

[37] C. W. von Keyserlingk, V. Khemani, and S. L. Sondhi, Absolute Stability and Spatiotemporal Long-Range Order in Floquet Systems, Phys. Rev. B 94, 085112 (2016).

[38] S. Choi, J. Choi, R. Landig, G. Kucsko, H. Zhou, J. Isoya, F. Jelezko, S. Onoda, H. Sumiya, V. Khemani, C. von
Keyserlingk, N. Y. Yao, E. Demler, and M. D. Lukin, Observation of Discrete Time-Crystalline Order in a Disordered Dipolar Many-Body System, Nature (London) 543, 221 (2017).

[39] J. Zhang, P. W. Hess, A. Kyprianidis, P. Becker, A. Lee, J. Smith, G. Pagano, I.-D. Potirniche, A. C. Potter, A. Vishwanath, N. Y. Yao, and C. Monroe, Observation of a Discrete Time Crystal, Nature (London) 543, 217 (2017).

[40] R. Moessner and S. L. Sondhi, Equilibration and Order in Quantum Floquet Matter, Nat. Phys. 13, 424 (2017).

[41] R. Nandkishore and A.C. Potter, Marginal Anderson Localization and Many-Body Delocalization, Phys. Rev. B 90, 195115 (2014).

[42] R. Nandkishore, Many-Body Localization and Delocalization in the Two-Dimensional Continuum, Phys. Rev. B 90, 184204 (2014).

[43] I. V. Gornyi, A. D. Mirlin, M. Müller, and D. G. Polyakov, Absence of Many-Body Localization in a Continuum, arXiv:1611.05895.

[44] R. Nandkishore, Many-Body Localization Proximity Effect, Phys. Rev. B 92, 245141 (2015).

[45] A. C. Potter and R. Vasseur, Symmetry Constraints on Many-Body Localization, Phys. Rev. B 94, 224206 (2016).

[46] W. De Roeck and F. Huveneers, Asymptotic Quantum Many-Body Localization from Thermal Disorder, Commun. Math. Phys. 332, 1017 (2014).

[47] W. De Roeck, F. Huveneers, M. Müller, and M. Schiulaz, Absence of Many-Body Mobility Edges, Phys. Rev. B 93, 014203 (2016).

[48] R. Nandkishore and S. Gopalakrishnan, Many Body Localized Systems Weakly Coupled to Baths, Ann. Phys. (Berlin) 529, 1600181 (2017).

[49] W. De Roeck and F. Huveneers, Stability and Instability towards Delocalization in MBL Systems, Phys. Rev. B 95, 155129 (2017).

[50] S. A. Parameswaran and S. Gopalakrishnan, SpinCatalyzed Hopping Conductivity in Disordered Strongly Interacting Quantum Wires, Phys. Rev. B 95, 024201 (2017).

[51] L. S. Levitov, Delocalization of Vibrational Modes Caused by Electric Dipole Interaction, Phys. Rev. Lett. 64, 547 (1990).

[52] A. L. Burin, Energy Delocalization in Strongly Disordered Systems Induced by the Long-Range Many-Body Interaction, arXiv:cond-mat/0611387.

[53] N. Y. Yao, C. R. Laumann, S. Gopalakrishnan, M. Knap, M. Müller, E. A. Demler, and M. D. Lukin, Many-Body Localization in Dipolar Systems, Phys. Rev. Lett. 113, 243002 (2014).

[54] Such low-temperature MBL is sometimes referred to as "asymptotic" localization [46]. Asymptotic localization is weaker than "infinite-temperature MBL" because it remains possible that rare "hot bubbles" $[47,49]$ may cause delocalization on exponentially long time scales. However, even asymptotic MBL is more than folk wisdom permits, and indeed is likely to be indistinguishable from "true MBL" [7] in laboratory experiments. 
[55] A. L. Efros and B. I. Shklovskii, Coulomb Gap and Low Temperature Conductivity of Disordered Systems, J. Phys. C 8, L49 (1975).

[56] G. L. Celardo, R. Kaiser, and F. Borgonovi, Shielding and Localization in the Presence of Long-Range Hopping, Phys. Rev. B 94, 144206 (2016).

[57] L. F. Santos, F. Borgonovi, and G. L. Celardo, Cooperative Shielding in Many-Body Systems with Long-Range Interaction, Phys. Rev. Lett. 116, 250402 (2016).

[58] J. Schwinger, Gauge Invariance and Mass. II, Phys. Rev. 128, 2425 (1962).

[59] S. Coleman, More About the Massive Schwinger Model, Ann. Phys. (N.Y.) 101, 239 (1976).

[60] D. Wolf and J. Zittartz, Physics of the Schwinger Model, Z. Phys. B 59, 117 (1985).

[61] W. Fischler, J. Kogut, and L. Susskind, Quark Confinement in Unusual Environments, Phys. Rev. D 19, 1188 (1979).

[62] T. Giamarchi, in Quantum Physics in One Dimension, International Series of Monographs (Clarendon Press, Oxford, 2004).

[63] To obtain the expression below for $H_{\text {int }}$, one can either Fourier transform, bosonize, then inverse Fourier transform, or bosonize and then integrate twice by parts.

[64] Ed. Witten (private communication).

[65] T. Giamarchi and H. J. Schulz, Anderson Localization and Interactions in One-Dimensional Metals, Phys. Rev. B 37, 325 (1988).

[66] I. V. Gornyi, A. D. Mirlin, and D. G. Polyakov, Electron Transport in a Disordered Luttinger Liquid, Phys. Rev. B 75, 085421 (2007).

[67] M. Müller, Purely Electronic Transport and Localization in the Bose Glass, Ann. Phys. (Berlin) 18, 849 (2009).

[68] V. Gurarie and J. T. Chalker, Some Generic Aspects of Bosonic Excitations in Disordered Systems, Phys. Rev. Lett. 89, 136801 (2002).

[69] I. L. Aleiner, B. L. Altshuler, and G. V. Shlyapnikov, A Finite-Temperature Phase Transition for Disordered Weakly Interacting Bosons in One Dimension, Nat. Phys. 6, 900 (2010).

[70] I. V. Gornyi, A. D. Mirlin, M. Müller, and D. G. Polyakov, Absence of Many-Body Localization in a Continuum, Ann. Phys. (Berlin) 529, 1600365 (2017).

[71] E. Abrahams, P. W. Anderson, D. C. Licciardello, and T. V. Ramakrishnan, Scaling Theory of Localization: Absence of Quantum Diffusion in Two Dimensions, Phys. Rev. Lett. 42, 673 (1979).

[72] F. D. M. Haldane, Luttinger Liquid Theory of OneDimensional Quantum Fluids. I. Properties of the Luttinger Model and Their Extension to the General 1D Interacting Spinless Fermi Gas, J. Phys. C 14, 2585 (1981).

[73] A. Imambekov, T. L. Schmidt, and L. I. Glazman, OneDimensional Quantum Liquids: Beyond the Luttinger Liquid Paradigm, Rev. Mod. Phys. 84, 1253 (2012).

[74] In a sense, we have traded a problem of rare regions containing very high-energy excitations, where perturbation theory is inapplicable, for a problem of rare regions containing far from ground-state fluctuations, where bosonization is inapplicable.
[75] M. Brenes, M. Dalmonte, M. Heyl, and A. Scardicchio, Many-Body Localization Dynamics from Gauge Invariance, arXiv:1706.05878.

[76] H. A. Fertig, Electromagnetic Response of a Pinned Wigner Crystal, Phys. Rev. B 59, 2120 (1999).

[77] E. Fradkin and S. H. Shenker, Phase Diagrams of Lattice Gauge Theories with Higgs Fields, Phys. Rev. D 19, 3682 (1979).

[78] V. Gurarie and J. T. Chalker, Bosonic Excitations in Random Media, Phys. Rev. B 68, 134207 (2003).

[79] S. Banerjee and E. Altman, Variable-Range Hopping through Marginally Localized Phonons, Phys. Rev. Lett. 116, 116601 (2016).

[80] W. Kohn and J. M. Luttinger, New Mechanism for Superconductivity, Phys. Rev. Lett. 15, 524 (1965).

[81] D. Fay and A. Layzer, Superfluidity of Low-Density Fermion Systems, Phys. Rev. Lett. 20, 187 (1968).

[82] S. Raghu, E. Berg, A. V. Chubukov, and S. A. Kivelson, Effects of Longer-Range Interactions on Unconventional Superconductivity, Phys. Rev. B 85, 024516 (2012).

[83] R. Nandkishore, R. Thomale, and A. V. Chubukov, Superconductivity from Weak Repulsion in Hexagonal Lattice Systems, Phys. Rev. B 89, 144501 (2014).

[84] P. W. Anderson, Theory of Dirty Superconductors, J. Phys. Chem. Solids 11, 26 (1959).

[85] I. V. Protopopov, W. W. Ho, and D. A. Abanin, The Effect of SU(2) Symmetry on Many-Body Localization and Thermalization, Phys. Rev. B 96, 041122 (2017).

[86] T. H. Hansson, V. Oganesyan, and S. L. Sondhi, Superconductors Are Topologically Ordered, Ann. Phys. (Amsterdam) 313, 497 (2004).

[87] S. Moroz, A. Prem, V. Gurarie, and L. Radzihovsky, Topological Order, Symmetry, and Hall Response of Two-Dimensional Spin-Singlet Superconductors, Phys. Rev. B 95, 014508 (2017).

[88] S. A. Parameswaran, S. A. Kivelson, R. Shankar, S. L. Sondhi, and B. Z. Spivak, Microscopic Model of Quasiparticle Wave Packets in Superfluids Superconductors Paired Hall StatesPhys. Rev. Lett. 109, 237004 (2012).

[89] T. Senthil and M. P. A. Fisher, Quasiparticle Density of States in Dirty High- $T_{c}$ Superconductors, Phys. Rev. B 60, 6893 (1999).

[90] T Senthil, M. P. A. Fisher, L. Balents, and C. Nayak, Quasiparticle Transport and Localization in High- $T_{c}$ Superconductors, Phys. Rev. Lett. 81, 4704 (1998).

[91] S. Vishveshwara, T. Senthil, and M. P. A. Fisher, Superconducting Metals, and “Insulators", Phys. Rev. B 61, 6966 (2000).

[92] X.-G. Wen, Quantum Field Theory of Many-Body Systems: From the Origin of Sound to an Origin of Light and Electrons (Oxford University Press on Demand, New York, 2004).

[93] M.P. A. Fisher, Vortex-Glass Superconductivity: A Possible New Phase in Bulk High-T $T_{c}$ Oxides, Phys. Rev. Lett. 62, 1415 (1989).

[94] D. S. Fisher, M. P. A. Fisher, and D. A. Huse, Thermal Fluctuations, Quenched Disorder, Phase Transitions, and 
Transport in Type-II Superconductors, Phys. Rev. B 43, 130 (1991).

[95] D. A. Huse, R. Nandkishore, F. Pietracaprina, V. Ros, and A. Scardicchio, Localized Systems Coupled to Small Baths: From Anderson to Zeno, Phys. Rev. B 92, 014203 (2015).

[96] R. Modak and S. Mukerjee, Many-Body Localization in the Presence of a Single-Particle Mobility Edge, Phys. Rev. Lett. 115, 230401 (2015).

[97] X. Li, S. Ganeshan, J. H. Pixley, and S. D. Sarma, ManyBody Localization and Quantum Nonergodicity in a Model with a Single-Particle Mobility Edge, Phys. Rev. Lett. 115, 186601 (2015).

[98] D.-L. Deng, S. Ganeshan, X. Li, R. Modak, S. Mukerjee, and J. H. Pixley, Many-Body Localization in Incommensurate Models with a Mobility Edge, Ann. Phys. (Berlin) 529, 1600399 (2017).

[99] M. Pretko, Generalized Electromagnetism of Subdimensional Particles: A Spin Liquid Story, Phys. Rev. B 96, 035119 (2017). 